\title{
Impact of comorbidity on survival after surgical resection in patients with stage I non-small cell lung cancer
}

\author{
Richard J. Battafarano, MD, PhDa \\ Jay F. Pic cirillo, MD \\ Bryan F. Meyers, MDa \\ Han-Shui Hsu, MDa \\ Tracey J. Guthrie, RN ${ }^{\mathrm{a}}$ \\ Joel D. Cooper, MD \\ G. Alexander Patterson, MD
}

Objective: As the mean age in patients with non-small cell lung cancer increases, the proportion of patients with serious comorbidity who are considered for surgical resection also increases. Patients with non-small cell lung cancer have been shown to have a higher burden of comorbidity than do patients with tumors of other sites, such as breast, prostate, colon, and head and neck. The goal of this review was to determine the impact of comorbidity on postoperative and long-term survival after surgical resection in patients with stage I non-small cell lung cancer.

Methods: A database analysis of our hospital's tumor registry included 451 patients who underwent surgical resection for pathologic stage I non-small cell lung cancer between January 1, 1994, and December 31, 1999. Comorbidity severity was prospectively determined with the Kaplan-Feinstein Index. Survival data were collected for each patient from the date of operation, with a mean duration of followup of 35.7 months. Bivariate statistics and Cox proportional hazards model analyses were used.

Results: The mean age was 64.4 years, and $249(55 \%)$ patients were male. The distribution of overall comorbidity severity was none, 142 (31.5\%); mild, 150 (33.3\%); moderate, 115 (25.5\%); and severe, $44(9.8 \%)$. The overall in-hospital mortality was $2.2 \%(n=10 / 451)$. There was a nonsignificant trend toward higher hospital mortality with greater comorbidity: none, $0.7 \%(n=1 / 142)$; mild, $3.3 \%(n=5 / 150)$; moderate, $0.9 \%(\mathrm{n}=1 / 115)$; and severe, $6.8 \%(\mathrm{n}=3 / 44, P=.055)$. Kaplan-Meier estimated survivals at 3 years for each level of comorbidity were as follows: none, $85.6 \%$; mild, $74.8 \%$; moderate, $68.8 \%$; and severe, $70.0 \%(P<.002)$. After adjustment for age, sex, T status, and tumor histologic type, the relative risks of death as a function of comorbidity were mild, 1.44 (95\% confidence interval $0.89-2.34$ ); moderate, 2.28 (95\% confidence interval 1.43-3.65); and severe; 1.94 (95\% confidence interval 1.023-3.70).

Conclusions: Comorbidity has a significant impact on survival after surgical resection of patients with stage I non-small cell lung cancer. These data may help to explain the lower than expected survival results for patients after surgical resection for stage I non-small cell lung cancer.

Adress for reprints: Richard J. Battafarano, MD, PhD, One Barnes-Jewish Plaza, 3107 Queeny Tower, St Louis, MO 63110-1013 (E-mail: battafarano@msnotes.wustl.edu).

J Thorac Cardiovasc Surg 2002;123:280-7

Copyright (C) 2002 by The American Association for Thoracic Surgery

$0022-5223 / 2002 \$ 35.00+0 \quad \mathbf{1 2 / 6 / 1 1 9 3 3 8}$

doi:10.1067/mtc. 2002.119338

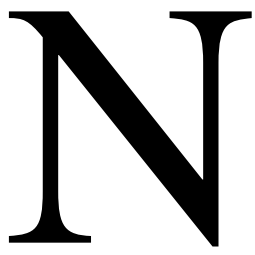

on-small cell lung cancer (NSCLC) is responsible for more than 120,000 deaths per year in the United States. ${ }^{1}$ Most patients are first seen with locally advanced or metastatic NSCLC that is unresponsive to chemotherapy and radiation therapy, and these individuals ultimately die of their disease. However, patients with a single focus of NSCLC that is amenable to complete surgical 


\section{TABLE 1. Organ systems examined in the modified KFI}

Cardiovascular system
Myocardial infarction
Angina or coronary artery disease
Congestive heart failure
Arrhythmia
Hypertension
Venous disease
Peripheral arterial disease
Respiratory system
Restrictive lung disease
Chronic obstructive pulmonary disease
Gastrointestinal system
Hepatic disease
Stomach or intestinal disease
Pancreatic disease
Renal system
End-stage renal disease
Endocrine system
Diabetes mellitus
Neurologic system
Stroke or cerebrovascular accident
Dementia
Paralysis
Neuromuscular disease
Psychiatric system
Rheumatologic system
Immunologic system
AlDS
Malignancy
Solid tumor
Leukemicit drugs and myeloma
Lymphoma
Substance abuse
Ilcohol
Body obesity
Pagher

resection have a more favorable prognosis. Actuarial 5-year survivals among patients with completely resected stage I and stage II NSCLC are 55\% to $75 \%$ and $35 \%$ to $45 \%$, respectively. ${ }^{2-5}$

Many patients with lung cancer have concurrent medical conditions (comorbidities) that significantly affect overall health. In addition to other tobacco-associated illnesses, such as chronic obstructive pulmonary disease, atherosclerotic cardiovascular disease, and head and neck cancer, patients may have diabetes mellitus, chronic renal insufficiency, or morbid obesity. These comorbidities often influence both treatment selection and survival after treatment. The purpose of this study was to examine the impact of patient comorbidity on survival after surgical resection of stage I NSCLC.
TABLE 2. Demographic features and treatment information for the $\mathbf{4 5 1}$ patients undergoing resection for stage I NSCLC

\begin{tabular}{lc}
\hline Gender (No.) & $249(55.2 \%)$ \\
Male & $202(44.8 \%)$ \\
Female & $64.4 \pm 10.5$ \\
Age (y, mean \pm SD) & \\
Comorbidity severity (No.) & $142(31.5 \%)$ \\
None & $150(33.2 \%)$ \\
Mild & $115(25.5 \%)$ \\
Moderate & $44(9.8 \%)$ \\
Severe & \\
Type of resection (No.) & $369(81.8 \%)$ \\
Lobectomy & $32(7.1 \%)$ \\
Wedge & $14(3.1 \%)$ \\
Bilobectomy & $12(2.7 \%)$ \\
Pneumonectomy & $12(2.7 \%)$ \\
Segmentectomy & $6(1.3 \%)$ \\
Lobectomy and wedge & $6(1.3 \%)$ \\
Sleeve lobectomy & \\
Histologic type (No.) & $209(46.3 \%)$ \\
Adenocarcinoma & $176(39.0 \%)$ \\
Squamous & $27(6.0 \%)$ \\
Large cell & $23(5.1 \%)$ \\
Bronchoalveolar & $16(3.6 \%)$ \\
Adenosquamous & \\
T status & $250(55.4 \%)$ \\
T1 & $201(44.6 \%)$ \\
T2 &
\end{tabular}

\section{Methods Population Under Study}

The Barnes-Jewish Hospital Cancer Registry and the Thoracic Surgery database were queried, and records of 480 patients who underwent surgical resection for stage I NSCLC from January 1, 1994, to December 31, 1999, were selected. Of these patients, 451 (94\%) had comorbidity severity scores determined prospectively at the time of diagnosis of NSCLC, and these patients form the population studied in this analysis.

The surgical pathology reports of all patients selected from this database query were individually reviewed, and the final pathologic staging was assigned according to the 1997 revisions in the International System for Staging Lung Cancer. ${ }^{5}$ Only patients with pathologically staged T1 N0 M0 (stage IA) or T2 N0 M0 (stage IB) NSCLC tumors were included in the analysis. Patients with carcinoid tumors, patients who had been previously treated for lung cancer, and patients who had received preoperative chemotherapy or radiation therapy were also excluded from this analysis.

Tumor recurrence, patient survival, and cause of death were determined for each patient. Follow-up information, including cause of death, was acquired within the last 6 months for all patients through clinic follow-up notes, direct patient or family contact, contact with the patient's primary care physician, and review of all death certificates. This study represents a secondary data analysis of a prospective cohort study. Approval for this study 


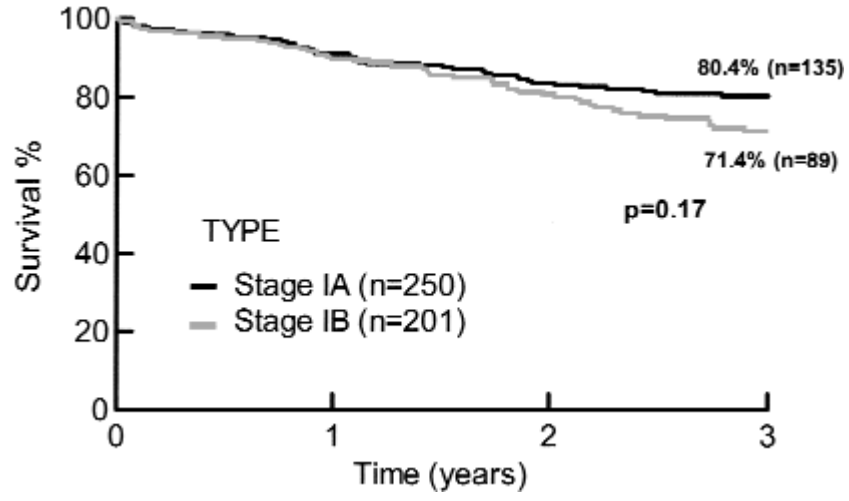

Figure 1. Three-year actuarial survival of the entire group stratified by stage IA and stage IB.

was granted from Washington University School of Medicine's human studies committee.

\section{Comorbidity Severity}

Since 1994, specifically trained cancer registrars have prospectively coded overall severity of comorbidity for patients with cancer treated at Barnes-Jewish Hospital, St Louis, Mo, with a modified version of the Kaplan-Feinstein Index (KFI). ${ }^{6}$ Minor modifications to the KFI were made to include diabetes mellitus and other comorbid conditions not present on the original KFI. The KFI classifies the pathophysiologic derangement of each comorbid ailment according to a four-category system $(0,1,2,3)$. An overall comorbidity score (none, mild, moderate, or severe) is determined on the basis of the number of ailments and their individual degrees of decompensation. The organ systems examined with the modified KFI are outlined in Table 1. The cancer registrars determined the comorbidity severity in their usual abstraction of the medical record. ${ }^{7}$

\section{Statistical Analysis}

Descriptive statistics were used to describe the patients' characteristics and outcomes. Normally distributed continuous data are expressed as mean \pm SD throughout. Medians with ranges are used when continuous data are not normally distributed. Categorical data are expressed as counts and proportions. The $\chi^{2}$ or Fisher exact test was used to analyze the categorical data. Kaplan-Meier (product-limit) graphs were used to demonstrate survival with time and freedom from recurrence of disease. Survival and eventfree survival comparisons between groups of patients were completed with the Mantel-Haenszel log rank test. Cox multivariate proportional hazards regression methods were used to discriminate independent risk factors for death after surgical resection for stage I NSCLC. Time to death after operation was selected as the primary outcome. The likelihood ratio method was used to determine hazard ratios, and the hazard ratio was used to approximate the relative risk.

All data analysis was performed with Systat (Systat 10.0 for Windows; SPSS Inc, Chicago, Ill).

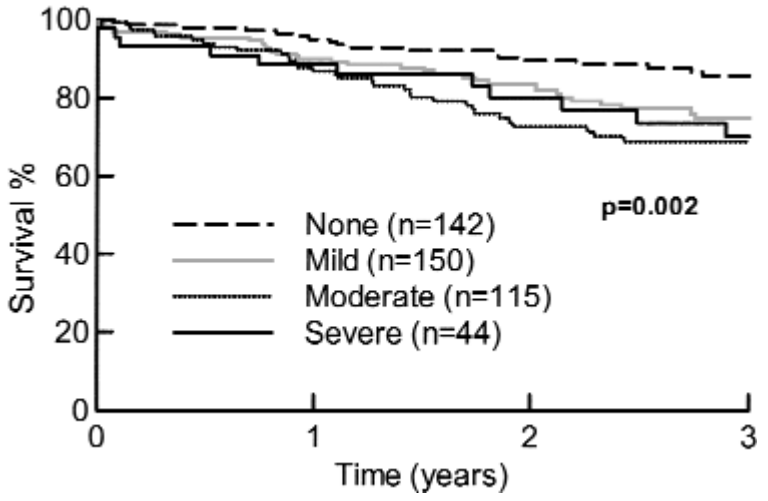

Figure 2. Three-year actuarial survival stratified by comorbidity severity.

\section{Results}

\section{Patient Demographic Characteristics}

Four hundred fifty-one patients with pathologic stage I NSCLC (T1 N0 M0 or T2 N0 M0) underwent surgical resection between January 1, 1994, and December 31, 1999 (Table 2). Two hundred forty-nine (55\%) patients were male and $202(45 \%)$ were female. The mean age was 64.4 \pm 10.5 years. The distribution of overall comorbidity severity was as follows: none, 142 (31.5\%); mild, 150 (33.3\%); moderate, 115 (25.5\%); and severe, 44 (9.8\%).

Two hundred fifty patients $(55 \%)$ had T1 tumors and 201 patients $(45 \%)$ had T2 tumors. Adenocarcinoma was the most common histologic variant $(46 \%)$. Four hundred nineteen patients $(93 \%)$ underwent anatomic resection. Most patients (82\%) underwent lobectomy for treatment of their NSCLC. However, 38 patients $(8 \%)$ required a more extensive operation to ensure complete resection. Forty-four patients $(10 \%)$ with $\mathrm{T} 1$ lesions underwent limited pulmonary resection by segmentectomy (3\%) or wedge excision $(7 \%)$.

\section{Survival}

Survival data were collected for each patient from the date of operation, with a mean duration of follow-up of 35.7 months. The 3-year actuarial survival for the entire group was $76.4 \%$. The 3-year actuarial survival of patients with T2 tumors $(71.4 \%)$ was lower than that of patients with T1 tumors $(80.4 \%)$. However, this difference was not statistically significant (Figure 1). When patients were stratified according to comorbidity severity, there was a significant relationship between comorbidity severity and survival $(P<$ .002 , Figure 2).

The association of comorbidity severity and survival was analyzed by multivariate Cox regression analysis. The additional variables of $\mathrm{T}$ status, age, gender, and tumor histologic type (Table 3) were included in the model. Patients 
TABLE 3. Results of multivariate analysis of prognostic factors influencing survival

\begin{tabular}{lcccc}
\hline Variable & No. & $\begin{array}{c}\text { Relative } \\
\text { risk }\end{array}$ & $\begin{array}{c}\text { Confidence } \\
\text { 95\% interval }\end{array}$ & $\begin{array}{c}\boldsymbol{P} \\
\text { value }\end{array}$ \\
\hline $\begin{array}{l}\text { Comorbidity severity } \\
\quad \text { None }\end{array}$ & 142 & 1.000 & - & - \\
$\quad$ Mild & 150 & 1.441 & $0.887-2.342$ & .140 \\
$\quad$ Moderate & 115 & 2.284 & $1.430-3.651$ & .001 \\
$\quad$ Severe & 44 & 1.944 & $1.023-3.699$ & .042 \\
T status & & & & \\
T1 & 250 & 1.000 & - & - \\
$\quad$ T2 & 201 & 1.365 & $0.957-1.944$ & .086 \\
Age (y) & 451 & 1.017 & $0.997-1.037$ & .088 \\
Gender & & & & \\
$\quad$ Female & 202 & 1.000 & - & - \\
$\quad$ Male & 249 & 1.190 & $0.995-1.120$ & .349 \\
Histologic type & & & & \\
$\quad$ Other & 242 & 1.000 & - & - \\
Adenocarcinoma & 209 & 0.921 & $0.646-1.312$ & .649 \\
\hline
\end{tabular}

with mild comorbidity severity had a slightly increased risk of death in the follow-up period relative to patients with a comorbidity severity of none, although this difference did not achieve statistical significance (relative risk 1.44, 95\% confidence interval 0.89-2.34, $P=.14)$. However, patients with moderate comorbidity severity (relative risk $2.28,95 \%$ confidence interval 1.43-3.65, $P=.001$ ) and severe comorbidity severity (relative risk $1.94,95 \%$ confidence interval 1.023-3.70, $P=.042$ ) were approximately twice as likely to die during the follow-up period as were patients with no comorbidity. There was no significant association of $\mathrm{T}$ status, age, gender, or tumor histologic type with survival.

\section{Causes of Death}

One hundred twenty-eight patients (28\%) died during the follow-up period (Table 4). Ten patients (2.2\%) died in the immediate postoperative period. Although there was a trend toward higher operative mortality among patients with more severe comorbidity, this did not achieve statistical significance $(P=.055)$. The proportion of patients who died from recurrent disease did not differ across comorbidity severity classification $(P=.92)$. However, the proportion of patients who died from causes other than recurrent lung cancer was significantly higher among patients with increasing comorbidity severity $(P<.001)$.

\section{Causes of Death Other than Recurrent Lung Cancer}

Of the 118 patients who died during the follow-up period, $68(57 \%)$ died of causes other than recurrent lung cancer (Table 5). The most common cause of death was cardiac disease, followed by death from a second malignancy.

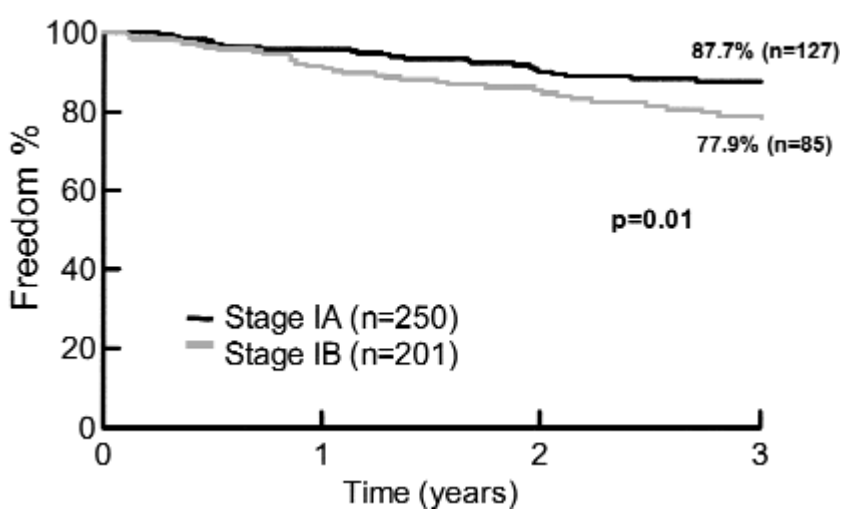

Figure 3. Three-year disease-free actuarial survival stratified by stage IA and stage IB.

Respiratory failure, cerebrovascular disease, sepsis, pulmonary embolism, motor vehicle crashes, and diabetes were also causes of death in this group of patients.

Fifty-two patients had new primary malignancies develop after resection of NSCLC (Table 6). The most common second malignancy encountered was NSCLC. Although it is often difficult to differentiate a second primary tumor from an isolated pulmonary metastatic focus, 13 of 23 second primary lung tumors had a different histologic type at the time of diagnosis. The remaining 10 patients had isolated contralateral tumors with no other evidence of disease. In addition, pathologic examination suggested a primary carcinoma rather than a contralateral hematogenous metastasis.

\section{Incidence of Recurrent Lung Cancer}

Seventy-one patients (15.7\%) had recurrent NSCLC during the follow-up period. The rates of recurrence as a function of comorbidity severity were as follows: none, $15.4 \%(\mathrm{n}=$ $22 / 142)$; mild, $16.7 \%(\mathrm{n}=25 / 150)$; moderate, $16.5 \%(\mathrm{n}=$ $19 / 115)$; and severe, $11.4 \%(\mathrm{n}=5 / 44, P=.85)$. The recurrence-free 3-year survival of patients with $\mathrm{T} 2$ tumors was significantly lower than that of patients with $\mathrm{T} 1$ tumors $(P$ $=.01$, Figure 3 ).

\section{Discussion}

Patients with NSCLC often have serious comorbidity at the time of diagnosis. In addition to atherosclerotic cardiovascular disease and chronic obstructive pulmonary disease, many of these patients also have chronic renal insufficiency, diabetes mellitus, a history of a previously treated malignancy, or morbid obesity. Because 5-year survival is a standard measure for the effectiveness of lung cancer treatment in this disease, it is critical to understand the impact of comorbidity on survival after the surgical treatment of NSCLC. 
TABLE 4. Causes of death for the $\mathbf{1 2 8}$ patients stratified by preoperative comorbidity score

\begin{tabular}{|c|c|c|c|c|c|}
\hline Preoperative comorbidity & None $(n=142)$ & Mild $(n=150)$ & Moderate $(n=115)$ & Severe $(n=44)$ & $P$ value \\
\hline Operative mortality & $1(0.7 \%)$ & $5(3.3 \%)$ & $1(0.9 \%)$ & $3(6.8 \%)$ & .055 \\
\hline Recurrent cancer & $16(11.3 \%)$ & $16(10.7 \%)$ & $14(12.2 \%)$ & $4(9.1 \%)$ & .922 \\
\hline Other & $12(8.5 \%)$ & $18(12.0 \%)$ & $31(26.9 \%)$ & $7(15.9 \%)$ & $<.001$ \\
\hline
\end{tabular}

TABLE 5. Causes of death of patients who died as a result of factors unrelated to resection or recurrence of the primary NSCLC

\begin{tabular}{lc}
\hline Cause of death & No. \\
\hline Cardiac failure & 18 \\
Other malignancy & 17 \\
Respiratory failure & 10 \\
Cerebrovascular accident & 5 \\
Sepsis & 4 \\
Pulmonary embolism & 3 \\
Motor vehicle crash & 3 \\
Diabetes & 3 \\
Other & 5 \\
Total & 68 \\
\hline
\end{tabular}

The importance of comorbidity in patients with lung and other cancers was initially identified by Feinstein and colleagues more that thirty years ago. ${ }^{8-13}$ The original goal of their research was to examine the impact of different cancer treatments on prognosis. However, they observed that a significant proportion of patients with localized cancer had poor outcomes, whereas other patients with more advanced stages had satisfactory outcomes. Careful review of the medical records demonstrated that patient-based factors of cancer-related symptom severity and comorbidity explained much of the discrepancy between TNM stage and patient outcome. ${ }^{14}$ Other investigators have confirmed the importance of comorbidity in the outcome of patients with cancers at other anatomic sites. ${ }^{15-21}$

In this analysis comorbidity severity had a significant negative impact on survival after surgical resection among patients with stage I NSCLC. After controlling for age, gender, $\mathrm{T}$ status, and tumor histologic type in a multivariate analysis, the relative risk of mortality as a function of comorbidity was 2 times higher among patients with moderate and severe comorbidity than among patients with no comorbidity. The higher risk of death was attributable to the comorbid conditions, because the proportion of patients who died of causes other than recurrent lung cancer was significantly higher among those with moderate and severe comorbidity.

The data from this analysis demonstrate the influence of comorbidity on survival in patients treated for stage I NSCLC and validate the ability of the modified KFI to strat-
TABLE 6. New primary malignancies after initial resection

\begin{tabular}{lr}
\hline Location of new primary tumor & No. \\
\hline Lung & 23 \\
Head and neck & 6 \\
Prostate & 5 \\
Gastric or esophageal & 3 \\
Bladder or kidney & 3 \\
Breast & 2 \\
Bone & 2 \\
Pancreas & 2 \\
Colon & 2 \\
Other & 4 \\
Total & 52 \\
\hline
\end{tabular}

ify comorbidity severity. These findings have important implications in the development of future clinical trials that attempt to evaluate the effectiveness of various therapies in the treatment of patients with NSCLC. If patients with moderate and severe comorbidity are to be included in these clinical trials, it is important to stratify the analysis according to comorbidity when reporting 5-year survivals, because these patients often die of causes other than recurrent lung cancer. An alternative, less desirable because as many as $35 \%$ of patients with stage I NSCLC have moderate or severe comorbidity, would be to exclude such patients from participation in these trials. The eligibility criteria for many current clinical trials include the assessment of performance status according to either the Karnofsky index or the Eastern Cooperative Oncology Group performance scale. These measures of performance status address the activity levels of individual patients and are often influenced by a patient's motivation and social support. In contrast, determination of comorbidity severity provides a more objective measure of a patient's physiologic reserve before the initiation of treatment.

Because the overall 5-year survival for patients with stage IB tumors (T2 N0 M0) is approximately $55 \%,{ }^{5}$ there has been increasing enthusiasm for including these patients in clinical trials to assess the efficacy of neoadjuvant (Intergroup S9900) or adjuvant (Intergroup 9633) chemotherapy. However, the data from this analysis demonstrated that most deaths in this group occurred as the result of 
causes other than recurrent lung cancer. In this analysis only $22 \%$ of patients with T2 tumors had recurrence and thus would potentially benefit from neoadjuvant or adjuvant chemotherapy. Because chemotherapy treatment has associated toxic side effects, patients with moderate or severe comorbidity severity might not ever be eligible for inclusion in these clinical trials, bringing the number of patients who could possibly benefit from this therapy even lower.

If chemotherapy treatment were able to reduce the recurrence rate from $22 \%$ to $11 \%$ (50\% risk reduction), then 9 patients would need to receive chemotherapy to prevent recurrence in 1 patient (according to the number to treat analysis). ${ }^{22}$ If chemotherapy treatment were only able to reduce the recurrence rate from $22 \%$ to $16.5 \%$ (25\% risk reduction), then 18 patients would need to receive chemotherapy to prevent recurrence in 1 patient. According to this analysis, demonstrating the efficacy of neoadjuvant or adjuvant chemotherapy in decreasing tumor recurrence for patients with stage I NSCLC will be difficult unless this therapy can be demonstrated to reduce the risk of recurrence by more than $50 \%$. The findings from this study have important implications for the design and accrual of these clinical trials.

The care management of patients with NSCLC remains a significant challenge. Many patients have significant comorbidity that adversely affects overall survival. Anatomic resection of stage I NSCLC results in excellent tumor-free survival and remains the standard form of therapy. Efforts to improve survival after treatment of NSCLC will need to be directed both toward reducing the incidence of recurrent disease and toward optimizing the management of coexisting medical illnesses.

\section{References}

1. Greenlee RT, Hill-Harmon ME, Murray T, Thun M. Cancer statistics, 2001. CA Cancer J Clin. 2001;51:15-36.

2. Martini N, Burt ME, Bains MS, McCormack PM, Rusch VW, Ginsberg RJ. Survival after resection of stage II non-small cell lung cancer. Ann Thorac Surg. 1992;54:460-5.

3. Ginsberg RJ, Rubinstein LV. Randomized trial of lobectomy versus limited resection for T1 N0 non-small cell lung cancer. Lung Cancer Study Group. Ann Thorac Surg. 1995;60:615-22.

4. Martini N, Bains MS, Burt ME, Zakowski MF, McCormack P, Rusch $\mathrm{VW}$, et al. Incidence of local recurrence and second primary tumors in resected stage I lung cancer. $J$ Thorac Cardiovasc Surg. 1995;109:120-9.

5. Mountain CF. Revisions in the International System for Staging Lung Cancer. Chest. 1997;111:1710-7.

6. Kaplan MH, Feinstein AR. The importance of classifying initial comorbidity in evaluating the outcome of diabetes mellitus. J Chronic Dis. 1974;27:387-404.

7. Piccirillo JF, Creech CM, Zequeira R, Anderson S, Johnston AS. Inclusion of comorbidity into oncology data registries. $J$ Registry Manage. 1999;26:66-70.

8. Feinstein AR. Symptomatic patterns, biologic behavior, and prognosis in cancer of the lung: practical application of Boolean algebra and clinical taxonomy. Ann Intern Med. 1964;61:27-41.

9. Feinstein AR. Symptoms as an index of biological behaviour and prognosis in human cancer. Nature. 1966;209:241-5.
10. Feinstein AR, Spitz H. The epidemiology of cancer therapy, I: clinical problems of statistical surveys. Arch Intern Med. 1969;123:171-86.

11. Feinstein AR, Pritchett JA, Schimpff CR. The epidemiology of cancer therapy, II: the clinical course: data, decisions, and temporal demarcations. Arch Intern Med. 1969;123:323-44.

12. Feinstein AR, Pritchett JA, Schimpff CR. The epidemiology of cancer therapy, III: the management of imperfect data. Arch Intern Med. 1969;123:448-61.

13. Feinstein AR, Pritchett JA, Schimpff CR. The epidemiology of cancer therapy, IV: the extraction of data from medical records. Arch Intern Med. 1969;123:571-90.

14. Feinstein AR. On classifying cancers while treating patients. Arch Intern Med. 1985;145:1789-91.

15. Neel HB 3rd, Taylor WF, Pearson GR. Prognostic determinants and a new view of staging for patients with nasopharyngeal carcinoma. Ann Otol Rhinol Laryngol. 1985;94:529-37.

16. Greenfield S, Aronow HU, Elashoff RM, Watanabe D. Flaws in mortality data: the hazards of ignoring comorbid disease. JAMA. 1988;260:2253-5.

17. Satariano WA. Comorbidity and functional status in older women with breast cancer: implications for screening, treatment, and prognosis. J Gerontol. 1992;47:24-31.

18. Peipert JF, Wells CK, Schwartz PE, Feinstein AR. Prognostic value of clinical variables in invasive cervical cancer. Obstet Gynecol. 1994;84:746-51.

19. Piccirillo JF, Wells CK, Sasaki CT, Feinstein AR. New clinical severity staging system for cancer of the larynx: five-year survival rates. Ann Otol Rhinol Laryngol. 1994;103:83-92.

20. Piccirillo JF, Feinstein AR. Clinical symptoms and comorbidity: significance for the prognostic classification of cancer. Cancer. 1996;77:834-42.

21. Pugliano FA, Piccirillo JF, Zequeira MR, Emami B, Perez CA, Simpson JR, et al. Clinical-severity staging system for oropharyngeal cancer: five-year survival rates. Arch Otolaryngol Head Neck Surg. 1997;123:1118-24.

22. Sackett DL, Haynes RB, Tugwell P. clinical epidemiology: a basic science for clinical medicine. Boston: Little Brown; 1991. p. 205-9.

\section{Discussion}

Dr John R. Roberts (Nashville, Tenn). This study is directed toward an important problem in cancer research: how patients' other diseases and illnesses may affect survival. This issue is particularly important for thoracic malignancies, because patients with lung and esophageal cancer often have other illnesses, such as emphysema, hypertension, and coronary disease. As oncologists, we are commonly told that surgery fails to cure early lung cancer $40 \%$ of the time, if we take Mountain's data, ${ }^{1}$ but we do not know from such studies what percentage of patients actually die of diseases other than recurrence of the malignancy. Obviously, treatments directed toward preventing cancer recurrence would not decrease the death rate from unrelated diseases.

Surgeons have been accustomed to using at least three types of illness scales in evaluating a patient's response to disease or treatments. Acute injury scales, such as the Acute Physiology and Chronic Health Evaluation system, attempt to measure the severity of an acute injury such as occurs in trauma. Oncologists are accustomed to using performance scales, such as the Karnofsky and Eastern Cooperative Oncology Group scales, and rating systems that assess a patient's functional capacity to work and to perform daily activities. These have been validated for treatment of patients with chemotherapy, but they only indirectly measure the effects of comorbid illnesses. Probably less common are comorbid index systems that measure two aspects of a patient's health, health irrespective of cancer and the impact of cancer on their 
health as measured by symptoms, such as the modified KFI described by Battafarano and coworkers.

I really have only two questions. First, Dr Battafarano, you took great care in measuring the comorbid status of patients with lung cancer. Do you also have data about other more commonly used performance scales in oncology, such as the Eastern Cooperative Oncology Group and Karnofsky scales? They should intersect somewhat with measures of comorbidity, and it would be interesting to see how those data vary.

Second, would not disease-free survival or perhaps freedom from recurrence get at the same issue that you have touched on in talking about comorbidity studies? That is, we want to know what fraction of patients actually die of the cancer to optimize cancer therapy, as opposed to being confused by the fraction of patients who die of other problems.

\section{Reference \\ 1. Mountain CF. Revisions in the International System for Staging Lung Cancer. Chest. 1997;111:1710-7.}

Dr Robert J. Ginsberg (Toronto, Ontario, Canada). We have to be careful about a few things. Only half the patients reached the 3 -year follow-up mark, so we do not have the usual 5-year assessment of recurrence.

In your article you did not discuss how unusual the number of noncancer deaths were in this series compared to most series, which usually quote a figure of $20 \%$ of deaths as noncancer deaths, whereas in your series the figure was $60 \%$. How do you explain that? Maybe things have changed.

I also would like you to address the fact that you are discussing patients with pathologic T2 tumors, suggesting that they are not candidates for, for instance, neoadjuvant therapy, whereas in the neoadjuvant protocols it is not pathologic staging that we discuss but clinical staging. We know from past experience at least that only $40 \%$ of patients with clinically staged T2 N0 disease survive 5 years, because half of them do not have T2 N0 disease according to pathologic examination.

The other area in your article was your discussion of clinical trials and the cost-benefit reality of clinical trials with respect to patient tolerance. If we can cure 1 in 10 of these patients by the addition of combined modality therapy, considering the fact there are 40,000 people in the United States who have this stage of disease, we are doing pretty well.

Dr Scott J. Swanson (Boston, Mass). I have a question about the respiratory failure group. Respiratory failure was the third highest noncancer cause of death. Do you have any details on that? Was it early or late, and was it related to preoperative forced expiratory volume in 1 second?

Dr Richard I. Whyte (Stanford, Calif). I noticed when reading the abstract that you took these patients during a 5-year period of time, and we are repeatedly hearing how patients who are having operations for lung cancer are getting older and sicker. Were you able to see any difference between the beginning of that 5-year study in 1994 and the end in 1999?

Dr Battafarano. With respect to Eastern Cooperative Oncology Group and Karnofsky performance scores, we did not specifically look at them in direct comparison with comorbidity severity scores. I think that one advantage of comorbidity severity scores is that they provide a more detailed analysis, rather than merely determining whether people are able to meet the demands of activities of daily living. However, I do not have direct comparisons between Eastern Cooperative Oncology Group scores or Karnofsky performance scores and comorbidity scores.

With respect to the incidence of recurrent lung cancer, it was approximately $16 \%$ across all four comorbidity severity determinations and did not differ among them. We plotted disease-free survival curves across comorbidity severity, but they did not show any differences. That is why we did not include them in the article.

Dr Ginsberg, you were saying that only half of the patients reached the 3-year follow-up mark and so we did not have 5-year survival data for this population. I think that this is a unique population. As you know, at Barnes we have a respiratory failure program, and that is where some of these patients' cancers were identified. I think that our population is therefore more heavily skewed with patients with moderate and severe comorbidity than the average clinical trial, from which many of these people would be excluded. More than half of all deaths resulted from causes other than recurrent lung cancer. I think that this is because we operated on patients with moderate and severe comorbidity, whereas in other series those patients have often been excluded or did not represent as high a proportion as in our population.

With respect to the chemotherapy, there are two issues there. One trial is sponsored by the Cancer and Leukemia Group B (CALGB), and it is an intergroup protocol in which they actually enrolled patients with T2 N0 tumors after pathologic staging, so that would be an adjuvant trial. The Bimodality Lung Oncology Trial includes people with clinically staged T2 N0 disease, which indeed may prove to be $\mathrm{T} 2 \mathrm{~N} 1$, and this would be a neoadjuvant trial. There is a difference, and I think that maybe these data would be more applicable to the CALGB-sponsored clinical trial, in which they are looking at adjuvant therapy.

I do not believe that this was a cost-benefit analysis. We mentioned in the article that if only $22 \%$ of patients with T2 N0 disease had recurrence and there was a $50 \%$ risk reduction from chemotherapy in the development of recurrent disease, then you would have to treat 9 patients to prevent 1 patient from getting recurrent lung cancer. If your risk reduction is only $25 \%$, you would have to treat 18 patients. I think that this is important because the number of patients that had recurrent lung cancer, at least in this series, was lower than we would normally expect from the Mountain data. ${ }^{1}$

Dr Swanson, you wondered about the respiratory failure afterward. I do not have all the specifics. A lot of it was from pneumonia. I do not know whether that was early, within the first 3 postoperative months, or was during the 3-year follow-up period. We do not have any specific data to look at correlation with forced expiratory volume in 1 second, although that would be important to know.

Dr Whyte, you asked whether the patients tended to be sicker later during that 5-year period. I could not tease out that information with just this limited analysis. However, we will look back at, for example, a cohort of patients from 1990 to 1995 and compare it with our cohort here, and that might be important.

\section{Reference}

1. Mountain CF. Revisions in the International System for Staging Lung Cancer. Chest. 1997;111:1710-7. 
Dr David J. Sugarbaker (Boston, Mass). In this cohort of patients during this period, did you exclude some patients, or is this your entire cohort of patients with stage I NSCLC?

Dr Battafarano. A total of 480 patients underwent resection, and 451 of them were included in this analysis.

Dr Sugarbaker. And 29 were excluded because they were unavailable for follow-up?

Dr Battafarano. No, they were excluded because they did not have comorbidity scores determined prospectively.

Dr Sugarbaker. You made a point about the risk adjustment. In terms of this category of patient with the addition of either adjuvant or neoadjuvant chemotherapy, given the impact here of your comorbidities, could you just address the feasibility of this situation?

Dr Battafarano. I think that in most clinical trial studies right now patients with moderate and severe comorbidity would be excluded from participation just on the basis of their comorbid illnesses. These patients represent a third of those with NSCLC, and we must either somehow stratify according to comorbidity or continue to exclude them from participation in trials.

\section{ON THE MOVE?}

Don't miss a single issue of the journal! To ensure prompt service when you change your address, please photocopy and complete the form below.

Please send your change of address notification at least six weeks before your move to ensure continued service. We regret we cannot guarantee replacement of issues missed due to late notification.

\section{JOURNAL TITLE:}

Fill in the title of the journal here.

\section{OLD ADDRESS:}

Affix the address label from a recent issue of the journal here.

\section{NEW ADDRESS:}

Clearly print your new address here.

Name

Address

City/State/ZIP

\section{COPY AND MAIL THIS FORM TO:}

Mosby

Subscription Customer Service

6277 Sea Harbor Dr

Orlando, FL 32887
OR FAX TO:

407-363-9661

N/M Mosby
OR PHONE:

800-654-2452

Outside the U.S., call

407-345-4000 